\title{
Carcinoma de células ceruminosas en perro
}

ESTEFANÍA FLORES P. ${ }^{1}$, M.V., GINO CATTANEO M.V., ANDRÉS BASTÍAS P., M.V., DENISE GRIMAU C., M.V., Ma ALEJANDRA SOTO, LIC. M.V., JULIO LARENAS², M.V., MgScVet., FEDERICO CIFUENTES, M.V., MgScVet., MIGUEL SEPÚLVEDA T.M.

1 Cirugía. Depto. Cs. Clínicas.

2 Histopatología. Depto Patología Veterinaria. Facultad de Cs. Veterinarias y Pecuarias, Universidad de Chile.

\begin{abstract}
CEROUMINOUS GLAND CELL CARCINOMA IN A DOG

The ear is divided into three parts: the external ear, middle ear, and internal ear. The external ear is a funnel-shaped structure that channels air vibrations to the tympanic membrane. It consists of the auricle (pinna) and the external ear canal (external acoustic meatus) that extend to the tympanic membrane. The structure of external ear canal is both cartilaginous and osseous. Some hair and many tubular ceruminous and sebaceous glands are present in the canal. Their secretions forms cerumen, a mucilaginous fluid containing brown granules. Two surgical techniques are available for the treatment of neoplasms of the external canal: the lateral resection, for small neoplasm's nodules of the canal; and the ablation of the ear canal, operation of choice when the neoplastic mass is deep and extensive, within the ear canal. It describes the case of a male dog, Cocker Spaniel, ten years old, patient treated in Surgery, Faculty of Veterinary and animal Sciences, University of Chile, with an episode of external otitis and a tumor that involved the right ear pinna and external right ear canal. It was decided proceeded taking samples for biopsy and subsequently to perform the removal of the external ear canal. The histopathologic diagnosis was ceruminous gland cell carcinoma.
\end{abstract}

Key words: External neoplasic otitis, ceruminous cell carcinoma.

\section{RESUMEN}

El oído se divide en tres partes; el oído externo, el medio y el interno. El oído externo es una estructura en forma de embudo, que canaliza las vibraciones del aire hacia la membrana timpánica, Se compone del pabellón auricular (pinna), y el canal auditivo externo (meato acústico externo) que se extiende hasta la membrana timpánica. Su soporte es oseo y cartilaginoso y presenta en su interior pelos y glándulas tubulares ceruminosas y sebaceas cutas secreciones forman el cerumen, fluido mucilaginoso que contiene granulos de color café. Existen dos técnicas quirúrgicas para el tratamiento de neoplasias del oído externo: la resección lateral para pequeños nódulos en el canal y la ablación del conducto, operación de elección cuando la masa neoplásica es profunda y extensa comprometiendo 
todo el canal. Se describe el caso de un perro macho, Cocker Spaniel, de diez años de edad, paciente tratado en Cirugía de la Facultad de Ciencias Veterinarias y Pecuarias de la Universidad de Chile por un cuadro de otitis externa y un tumor que comprometía la cara interna del pabellón auricular y el conducto auditivo externo derecho. Se procedió a la toma de muestra para biopsia y posteriormente a la extracción del pabellón auricular, y ablación del conducto auditivo externo. El diagnóstico histopatológico fue adenocarcinoma de células ceruminosas.

Palabras clave: Otitis neoplasica externa, adenocarcinoma de células ceruminosas.

\section{INTRODUCCIÓN}

El oído externo está integrado por el pabellón de la oreja y el canal auditivo externo y varía en tamaño y forma entre las diferentes razas caninas, siendo el cartílago auricular quien determina la apariencia del pabellón auricular (Fossum 1999, Evans y deLahunta 2002).

Las características anatómicas del oído externo, para las diversas razas, pueden predisponer al desarrollo de ciertas patologías; por ejemplo, en las orejas largas y de pelo largo, la presencia de humedad ambiental, con el calor corporal, puede actuar como factor predisponente a las otitis externa.

La elección correcta del tratamiento es imprescindible, ya que un tratamiento inadecuado puede ser la causa de la pérdida de la audición del paciente, aumentar la magnitud del cuadro e incluso, en casos más severos, contribuir al desarrollo posterior de una neoplasia.

Se define la otitis externa como la inflamación del canal auditivo. Sus causas son numerosas, pudiendo ser divididas en predisponentes, primarias, secundarias $\mathrm{y} / \mathrm{o}$ perpetuantes. Los errores en el reconocimiento correcto de una o más de estas causas pueden llevar al fracaso terapéutico (Scott, Miller y Griffin 2001).

La otitis externa crónica puede ser el resultado de una neoplasia ótica, o la otitis puede ser un factor predisponente en la formación de la neoplasia. Los Cocker Spaniel tienen una incidencia muy alta de neoplasias benignas y malignas y de otitis externas. Dentro de las neoplasias predominan los tumores de la piel y de las estructuras anexas del oído. Los tumores benignos en los perros comprenden el adenoma de las glándulas sebáceas, el tumor basocelular, pólipos, el adenoma de las células ceruminosas y papilomas. Las neoplasias malignas en perros y gatos incluyen el adenocarcinoma de las células ceruminosas, el carcinoma indiferenciado y el carcinoma epidermoide. Los adenocarcinomas de las células ceruminosas son los tumores que se diagnostican con más frecuencia en el conducto auditivo de los perros y gatos; sin embargo, un estudio ha comprobado que el carcinoma epidermoide surge con la misma incidencia en el gato (Radlinsky y Mason, 2002).

Las neoplasias de las células ceruminosas son típicas de animales viejos y suelen aparecer en una sola oreja. Los signos clínicos incluyen grados variables de sacudidas de cabeza y rasquido de la oreja, otorrea, mal olor, frecuentemente otitis bacteriana externa secundaria e incluso hemorragia intermitente de la oreja afectada (Scott, Miller y Griffin 2001). Estos signos a menudo están presentes por meses o años antes de que el animal sea llevado al veterinario para su tratamiento (Vail y Withrow, 2007).

\section{DESCRIPCIÓN DEL CASO}

\section{Reseña}

Un canino macho, de raza Cocker Spaniel, de diez años de edad y $15,4 \mathrm{~kg}$ de peso, fue llevado a la consulta del Servicio de Cirugía de la Facultad de Ciencias Veterinarias y Pecuarias de la Universidad de Chile, debido a un cuadro de otitis externa unilateral. El paciente, además, presentaba varios tumores que abarcaban parte de la cara interna del pabellón auricular y la entrada del conducto auditivo del oído derecho (Figura 1).

\section{Anamnesis}

La propietaria refirió que el animal había sufrido de tres episodios de otitis externa durante el transcurso del año anterior, siendo tratado en las tres ocasiones mediante administración de antibióticos, cuyo nombre y ritmo horario no re- 


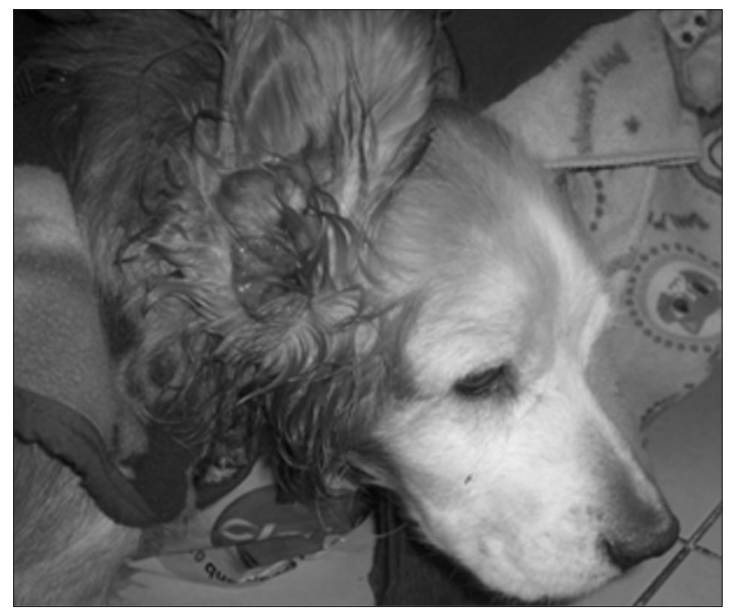

Figura 1. Masa de tumores que abarcaban parte de la cara interna del pabellón auricular a la entrada del conducto auditivo del oído derecho.

cordaba, no obteniendo resultados. Sin embargo, durante la administración del tratamiento, los tumores comenzaron a aumentar de tamaño.

\section{Examen físico}

El paciente presentaba tumoraciones que comprometían la cara interna del pabellón auricular en la proximidad de la entrada del meato auditivo externo derecho. Los tumores eran de consistencia firme, ulcerados y con secreción purulenta.

\section{Exámenes complementarios}

Se procedió a la toma de muestra de sangre para hemograma y perfil bioquímico y se recetó Amoxicilina $250 \mathrm{mg}$ (Biotivet ${ }^{\circledR}$, Laboratorios Chile) en dosis de $300 \mathrm{mg}$ cada 12 horas y limpiezas de la zona con clorhexidina al $2 \%$.

Los resultados de los exámenes arrojaron alteraciones en la bilirrubina total $(0,67 \mathrm{mg} / \mathrm{dl}$, valor de referencia $0,1-0,5 \mathrm{mg} / \mathrm{dl}$ ), asociados a la bilirrubina conjugada $(0,56 \mathrm{mg} / \mathrm{dl}$, valor de referencia 0 - 0,12 $\mathrm{mg} / \mathrm{dl})$, en la albúmina $(2,4 \mathrm{~g} / \mathrm{dl}$, valor de referencia $2,6-3,3 \mathrm{~g} / \mathrm{dl})$, globulinas $(4,6$ $\mathrm{g} / \mathrm{dl}$, valor de referencia $2,7-4,4)$ y en la relación albúmina/globulina $(0,52$, valor de referencia 0,59 - 1,11). Niveles elevados de Bilirrubina conjugada sugieren enfermedad hepática.

\section{Prediagnóstico}

Ante la presencia de un cuadro de etiología neoplásica y teniendo en consideración la

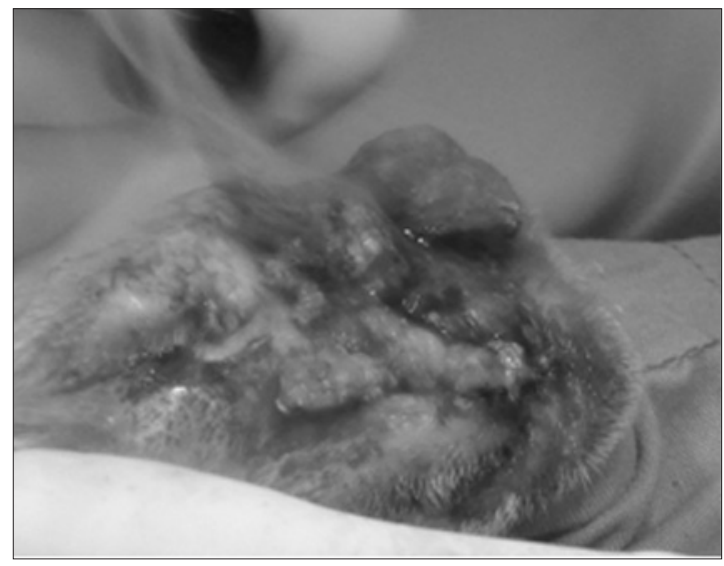

Figura 2. Toma de biopsia incisional. Se observan zonas ulceradas y pigmentadas de la neoplasia.

complejidad de la cirugía en la zona auricular y los tipos de neoplasias que se desarrollan en ella, la primera decisión a tomar para este caso fue la conveniencia de realizar una biopsia incisional preoperatoria para prediagnosticar el tipo de neoplasia presente, y así tener un elemento mas objetivo para determinar el tipo de tecnica quirurgica y los márgenes de seguridad para su extracción definitiva.

\section{Protocolo anestésico para biopsia insicional}

Para la extracción de las biopsias, el paciente fue anestesiado con un protocolo compuesto por premedicación con atropina $(0,04 \mathrm{mg} / \mathrm{kg}, \mathrm{IM}) \mathrm{y}$ acepromacina $(0,02 \mathrm{mg} / \mathrm{kg}, \mathrm{IM})$. La inducción se realizó mediante la administración a efecto de Tiopental sódico al $2 \%$.

\section{Biopsia incisional}

Se decidió extraer muestras para biopsia desde la cara interna del pabellón auricular (Figura 2). Las muestras fueron fijadadas en formalina y enviadas para su estudio histopatológico. Las muestras fueron enviadas al servicio de Anatomía Patológica de la Facultad de Ciencias Veterinarias y Pecuarias de la Universidad de Chile.

\section{Diagnóstico histopatológico}

Adenocarcinoma complejo de células ceruminosas (Figuras 3 y 4). Analizando los riesgos la magnitud de la cirugía oncológica necesaria para intentar una remisión completa, y el pronóstico de la neoplasia que afectaba al paciente se determinó realizar como tratamiento quirúrgico 
la ablación del conducto auditivo externo y tejidos vecinos que fuera necesario para dejar la zona sin tumor visible a simple vista.

Se observa tejido neoplásico mal delimitado, focalmente invasivo, compuesto por células de origen epitelial que se disponen en lóbulos separados por estroma fibrovascular grueso multifocalmente edematoso. Las células neoplásicas son pleomórficas, en la periferia de los lóbulos presentan escaso citoplasma, bordes angulares y núcleos eucromáticos ovalados a circulares con uno a tres nucléolos prominentes. Algunas de las células presentan citoplasma abundante, eosinofílico, finamente vacuolado, con núcleos redondos de ubicación central. Se observan dos a tres figuras mitóticas por campo de alto aumento, focos extensivos de necrosis, infiltrado inflamatorio polimorfonuclear neutrófilo y abundante irrigación.

\section{ABLACIÓN DEL CONDUCTO AUDITIVO EXTERNO}

\section{TÉCNICA ANESTÉSICA}

\section{Premedicación}

El protocolo anestésico utilizado incluyó atropina $(0,04 \mathrm{mg} / \mathrm{kg}$, im.), para antagonizar la posibilidad de una bradicardia o hipotensión secundaria al estímulo vagal provocado por el procedimiento en el oído, y debido al carácter inquieto del paciente, acepromacina $(0,02 \mathrm{mg} /$ $\mathrm{kg}$, im.) como tranquilizante, utilizándose la dosis mínima recomendada debido a la edad del paciente. Se incluyó como analgésico tramadol ( $2 \mathrm{mg} / \mathrm{kg}$ im.), debido a lo doloroso del procedimiento. Luego de la premedicación se procedió a canular la vena cefálica con una bránula de 22 $\mathrm{G}$, que se conectó a un set de administración de fluidos con un suero fisiológico. La inducción se realizó mediante la administración a efecto de tiopental sódico al $2 \%$. Una vez realizada la inducción se reguló el paso del suero fisiológico a una velocidad de $10 \mathrm{ml} / \mathrm{kg} /$ hora. Luego de esto se intubó y se conectó aun circuito anestésico de tipo circular, administrando 1 litro de oxígeno por minuto. Posteriormente, se conecto un capnógrafo y un monitor multiparámetros para la monitorización durante la anestesia general. Las variables fisiológicas monitoreadas fueron: frecuencia cardiaca, frecuencia respiratoria, presión arterial sistólica, temperatura, dióxido de carbono espirado y saturación de oxígeno arterial. La mantención se realizó con isoflurano, entregándose a concentraciones entre 2,5 a $1 \%$, a lo
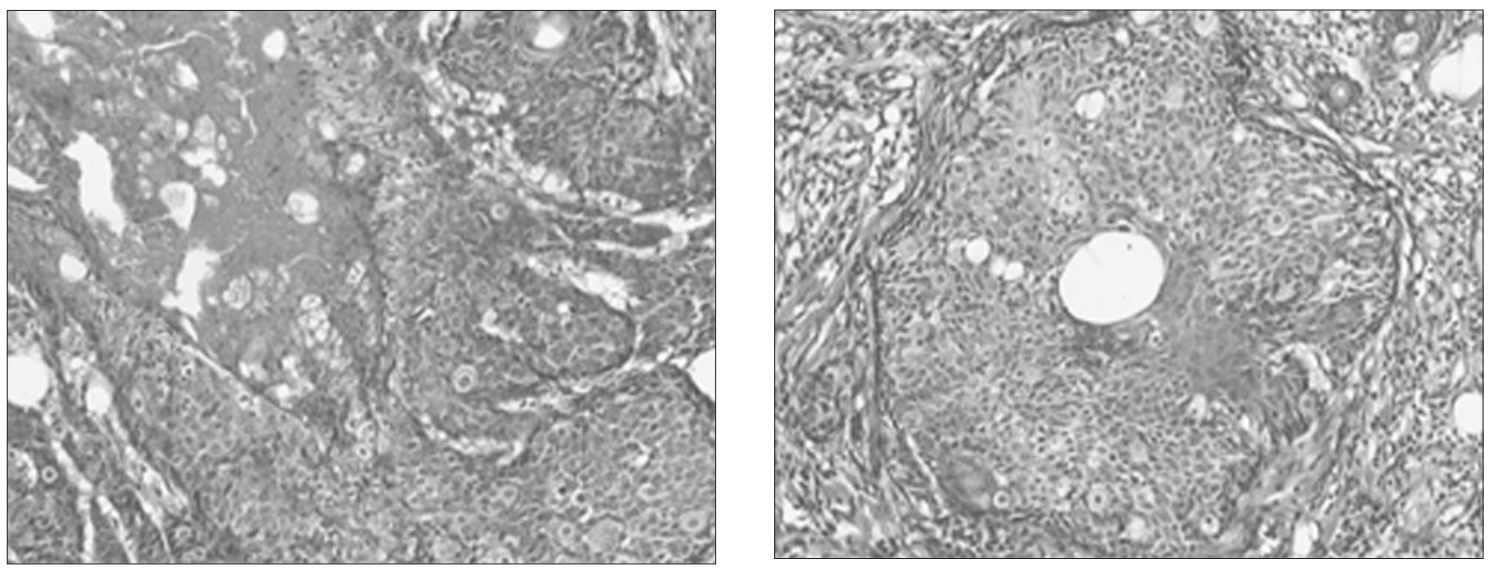

Figiguras 3 y 4. 40X Se observa tejido neoplásico mal delimitado, focalmente invasivo, compuesto por células de origen epitelial que se disponen en lóbulos separados por estroma fibrovascular grueso multifocalmente edematoso. Las células neoplásicas son pleomórficas, en la periferia de los lóbulos presentan escaso citoplasma, bordes angulares y núcleos eucromáticos ovalados a circulares con uno a tres nucléolos prominentes. Algunas de las células presentan citoplasma abundante, eosinofílico, finamente vacuolado, con núcleos redondos de ubicación central. Se observan dos a tres figuras mitóticas por campo de alto aumento, focos extensivos de necrosis, infiltrado inflamatorio polimorfonuclear neutrófilo y abundante irrigación. 
largo de la cirugía. Los parámetros fisiológicos antes mencionados se mantuvieron durante todo el procedimiento dentro de los rangos considerados normales para la especie, no registrándose efectos secundarios ni complicaciones.

Se realizó un bloqueo anestésico del nervio auricular mayor y del auriculotemporal, con bupivacaina al 0,5\%, para aportar una mayor analgesia durante la cirugía y en el postoperatorio inmediato.

\section{TÉCNICA QUIRÚRGICA}

Una vez depilados ambos lados del pabellón auricular derecho, el paciente fue colocado en la mesa de operaciones en decúbito lateral izquierdo. Se desinfectó la zona operatoria con alcohol yodado y se aisló el campo operatorio mediante paños de campo esteriles colocados alrededor de la oreja.

\section{Abordaje}

Se realizaron dos incisiones en la piel, paralelas al canal auditivo externo en su trayectio vertical, hasta un punto definido debajo del canal auditivo horizontal. Ambas incisiones fueron conectadas ventralmente y mediante disección roma y aguda se reflejó un colgajo de piel, exponiendo la pared cartilaginosa lateral del canal auricular vertical. Se resecó la mitad distal del colgajo de cartílago y se extrajo el colgajo cutáneo.

Luego se seccionó el canal vertical hasta alcanzar el nivel del canal horizontal, reflejando un colgajo de cartílago y se escindió la inserción del canal horizontal al meato acústico externo, luego con una cureta se removió el tejido adherido al meato acústico externo, se colocó un drenaje y se suturaron los tejidos subcutáneos y el tegumento.

Finalmente se procedió a la extracción del pabellón auricular, ligando previamente sus arterias y venas.

\section{POST OPERATORIO Y EPICRISIS}

Se indicó limpieza local con agua oxigenada de 10 vol, dos veces al día, seguida de aplicación de povidona yodada en los puntos de sutura. Se indicó protección continua día y noche con collar isabelino. Los puntos se extrajeron a los 10 días, con una buena reparación por primera intención.

\section{DISCUSIÓN}

La enfermedades del oído en general tienen una ubicación protegida de la vista en sus inicios, por lo que el diagnostico precoz es muy raro; y al avanzar no constituyen un peligro de muerte inminente por lo que son subdiagnosticadas y solo descubiertas cuando el compromiso local es muy manifiesto. Esa noción de "benignidad" en las neoplasias alojadas en el conducto es bastante errónea, y posiblemente se fundamenta en la falsa seguridad de aislamiento de la lesión que dan las estructuras cartilaginosas que limitan y dan estructura sólida al conducto auditivo externo. El crecimiento de neoformaciones en el interior del conducto puede ser limitado por un tiempo, pero en las sucesivas duplicaciones termina sobrepasando las barreras cartilaginosas y si además son celulas malignas, tienen una especial capacidad para invadir los tejidos circundantes, impidiendo al cirujano generar limites claros para la escisión del tumor con bordes libres de tejido neoplásico. Eso significa que no es posible asegurar la completa extracción quirúrgica, ni anticipar la posibilidad de recidiva y menos aun estimar el tiempo libre de tumor que se pueda obtener.

La realización de una bipsia incisional, previa a la cirugía es un tema digno de analizar:

$1^{\circ}$. La lógica elemental dicta que no se debe realizar si la "masa" a biopsiar es de un tamaño tal, que será igual la cirugía de extracción completa que la parcial; en cuyo caso mejor es realizar una cirugía oncológica, con márgenes libres de tumor, como primera acción, enviando la "masa" extraída completa al patólogo. Se pedirá identificación de las células de origen y estudio de márgenes para saber si fue extraída totalmente o hay crecimientos microscópicos que anticipen la recidiva y la generalización. Es el caso de masa bien circunscritas en el conducto auditivo externo, no en este caso.

$2^{\circ}$. También es de sentido común no realizarla si el resultado no va a cambiar la decisión de operar la neoplasia, cuando el dueño declara que realizará la cirugía sin importar si sea benigna o cancerosa. En ese caso también se 
enviará el neo completo, extraído con bordes oncológicos a biopsia posterior a la operación de extracción.

$3^{\circ}$. En este caso la biopsia se realizó para dar a su propietaria más elementos de decisión, para poder anticipar la extensión de la intervención, que por tratarse de una neo cancerosa, comprendería todo el oído externo, una cirugía mutilante en la que es necesario anticipar que alterará considerablemente la apariencia del paciente, como forma de tener más posibilidades de una más prolongada sobrevida libre de cáncer.

\section{REFERENCIAS}

1.- EVANS H, DELAHUNTA A. 2002. Disección del perro. $4^{\mathrm{a}}$ ed. McGraw-Hill Interamericana. México, D.F. $384 \mathrm{p}$.

2.- FOSSUM T. 1999. Cirugía en animales pequeños. Inter-Médica S.A.I.C.I. Buenos Aires, Argentina. 1300 p.

3.- RADLINSKY MG, MASON DE. 2007. Enfermedades del Oído. In: Ettinger, S.J.; Feldman, E.C. Tratado de Medicina Interna: Enfermedades del Perro y el Gato Volumen 2. 6a ed. Elsevier. Madrid, España. pp 1168-1186.

4.- SCOTT D, MILLER W, GRIFFIN C. 2001. Small Animal Dermatology. $6^{\mathrm{a}}$ ed. Saunders. Philadelphia, Penssylvania. $1526 \mathrm{p}$.

5.- VAIL D, WITHROW S. 2007. Tumors of the Skin and Subcutaneous Tissues. In: Small Animal Clinical Oncology. 4a ed. Saunders. United States. pp. 375-401. 